\title{
The effect of physiological concentrations of bile acids on the in vitro growth of Mycobacterium tuberculosis
}

\author{
D Epstein, K Mistry, A Whitelaw, G Watermeyer, K E Pettengell
}

Background. Intestinal tuberculosis occurs mainly in the terminal ileum and caecum, where the concentration of bile acids is lowest, and rarely in the upper digestive tract.

Objectives. We examined the effect of physiological concentrations of bile acids on the in vitro growth of Mycobacterium tuberculosis (MTB).

Methods. The 4 major bile acids, lithocolic acid, cholic acid, deoxycholic acid and chenodeoxycholic acid, were added to individual Lowenstein-Jensen (LJ) culture media at physiological concentrations. A combined LJ medium was also prepared using all 4 bile acids. These were double-diluted 4 times by the addition of LJ media. Each culture medium was inoculated with the H37Rv strain of MTB and incubated at $37^{\circ} \mathrm{C}$ for 8 weeks. MTB growth was measured at 2 and 8 weeks in a semiquantitative fashion using cutoffs of $>5,>10,>20,>100$ colony-forming units.

Results. All lithocolic acid cultures showed uninhibited TB growth at 2 and 8 weeks. Chenodeoxycholic acid, deoxycholic acid and cholic acid alone, and in combination, showed concentrationdependent inhibition of MTB growth at 2 and 8 weeks. Four cultures were lost to contamination.

Conclusions. Certain bile acids alone and in combination, at physiological concentrations, inhibit the growth of MTB in vitro. This might explain why intestinal $\mathrm{TB}$ occurs in the ileocaecum in the majority of cases and why gallbladder TB is very rare.

S Afr Med J 2012;102(6):522-524.
Intestinal tuberculosis (ITB) involves the ileocaecum in the majority of cases with upper gastrointestinal tract tuberculosis accounting for less than $3 \%$ of all cases. ${ }^{1}$ Gallbladder $\mathrm{TB}$, in particular, is very rare, with few cases reported in the literature. ITB results from haematogenous spread or spread from contiguous lymph nodes or swallowed infected sputum. ${ }^{2}$ Why ITB preferentially affects the ileocaecum is unclear, but proposed mechanisms include bowel stasis in the terminal ileum, due to the ileocaecal valve, and the high density of lymphoid tissue found in the terminal ileum.

Bile acids are the major constituent of bile and the primary bile acids, cholic and chenodeoxycholic acid, and secondary bile acids, lithocholic and deoxycholic acid, make up more than $95 \%$ of the bile acid pool. Bile acids have a number of biological effects, including generation of reactive oxygen species, a detergent action on lipidcontaining cell membranes and antimicrobial effects. Enterohepatic circulation of bile acids results in efficient reabsorption of bile acids, resulting in a concentration gradient between proximal small intestine and terminal ileum. Only $10 \%$ of bile acids escape reabsorption and studies have shown that the bile acid concentration in the caecum is below the micellisation concentration. ${ }^{3}$ Could the high concentration of bile acids in the gallbladder and the proximal intestine be

Division of Gastroenterology, Department of Medicine, University of Cape Town and Groote Schuur Hospital, Cape Town

D Epstein, MB ChB, DCH (SA), FCP (SA), Cert Gastroenterol (SA)

G Watermeyer, MB ChB, FCP (SA), Cert Gastroenterol (SA)

University College Medical School, London, UK

K Mistry, BSc, MB BS

National Health Laboratory Service, Groote Schuur Hospital and Division of Medical Microbiology, University of Cape Town

A Whitelaw, MB BCh, FC (Path) SA, MSc

Parklands Hospital, Durban

K E Pettengell, MD, FCP, FRCP, MB ChB, MRCP (UK) protective and explain the anatomical distribution of ITB?

We determined the effect of physiological concentrations of bile acids on the in vitro growth of Mycobacterium tuberculosis (MTB).

\section{Methods}

The 4 major bile acids, lithocolic acid, cholic acid, deoxycholic acid and chenodeoxycholic acid (all supplied by Sigma Aldrich, SA) were selected for study. Individual bile acids were added to standard Lowenstein-Jensen (LJ) media to achieve physiological concentrations, ${ }^{4}$ as shown in Table 1. A fifth LJ-bile acid solution was prepared using all 4 bile acids. Due to difficulties in solubilising the combination of 4 bile acids at physiological concentrations, the concentration of each had to be reduced by $50 \%$ in this $\mathrm{LJ}$ slope.

Each 'physiological' LJ-bile acid solution was subsequently doublediluted 4 times with pure LJ solution, to achieve a decreasing concentration of bile acid in the culture medium. A standard inoculum ( $0.5 \mathrm{McFarland}$ ) of MTB H37Rv was inoculated onto each LJ slope in triplicate, as well as onto LJ slopes with no bile salts added. The slopes were incubated at $37^{\circ} \mathrm{C}$ for 8 weeks. Mycobacterial growth was measured at 2 and 8 weeks in a semiquantitative fashion using cut-offs of $>5,>10,>20,>100$ colony-forming units (CFUs) per slope.

\section{Results}

There were $>100$ CFUs of typical MTB colonies on the control slopes at both 2 and 8 weeks. Similarly, all lithocolic acid cultures showed growth of $>100$ CFUs at 2 and 8 weeks.

Three of the bile acids alone (chenodeoxycholic acid, deoxycholic acid and cholic acid) and the combination of all 4 showed inhibition of growth at 2 and 8 weeks. Chenodeoxycholic acid appeared to be the most active of the bile salts, based on the results at 8 weeks, with inhibition of growth even at a 5 -fold dilution $(1.375 \mathrm{mg} / \mathrm{ml})$. As can be seen from Figs 1 - 4, a concentration-dependent effect was evident for the above bile acids alone and in combination. There was complete inhibition of growth of MTB at physiological and subphysiological concentrations of the above 3 bile salts at both 2 and 8 weeks. Results were largely reproducible across all three experiments.

Four cultures were lost to contamination. These included a deoxycholic acid culture at $8 \mathrm{mg} / \mathrm{ml}$ and 3 cholic acid cultures with the following concentrations: $16 \mathrm{mg} / \mathrm{ml}, 8 \mathrm{mg} / \mathrm{ml}$ and $4 \mathrm{mg} / \mathrm{ml}$. 


\section{RESEARCH}

Table 1. Dilution protocol of bile acids in Lowenstein-Jensen medium

\begin{tabular}{llllll}
\hline & \multicolumn{5}{c}{ Lowenstein-Jensen medium with bile acid additives } \\
\cline { 2 - 6 } & $\begin{array}{l}\text { Lithocolic acid } \\
(\mathbf{m g} / \mathbf{m l})\end{array}$ & $\begin{array}{l}\text { Cholic acid } \\
(\mathbf{m g} / \mathbf{m l})\end{array}$ & $\begin{array}{l}\text { Chenodeoxycholic } \\
\text { acid }(\mathbf{m g} / \mathbf{m l})\end{array}$ & $\begin{array}{l}\text { Deoxycholic acid } \\
(\mathbf{m g} / \mathbf{m l})\end{array}$ & $\begin{array}{l}\text { Combined bile acids } \\
\text { in physiological ratio } \\
(\mathbf{m g} / \mathbf{m l})\end{array}$ \\
\cline { 2 - 6 } Dilution 1 & 2 & 32 & 22 & 12 & - \\
Dilution 2 & 1 & 16 & 11 & 6 & 35 \\
Dilution 3 & 0.5 & 8 & 5.5 & 3 & 17.5 \\
Dilution 4 & 0.25 & 4 & 2.75 & 1.5 & 8.75 \\
Dilution 5 & 0.125 & 2 & 1.375 & 0.75 & 2.375 \\
Dilution 6 & - & - & - & - & 2.188 \\
Bil acid physiological & {$[1.5 \pm 1.4]$} & {$[18.3 \pm 12.4]$} & {$[11.5 \pm 4.9]$} & & \\
concentration based on & & & & &
\end{tabular}

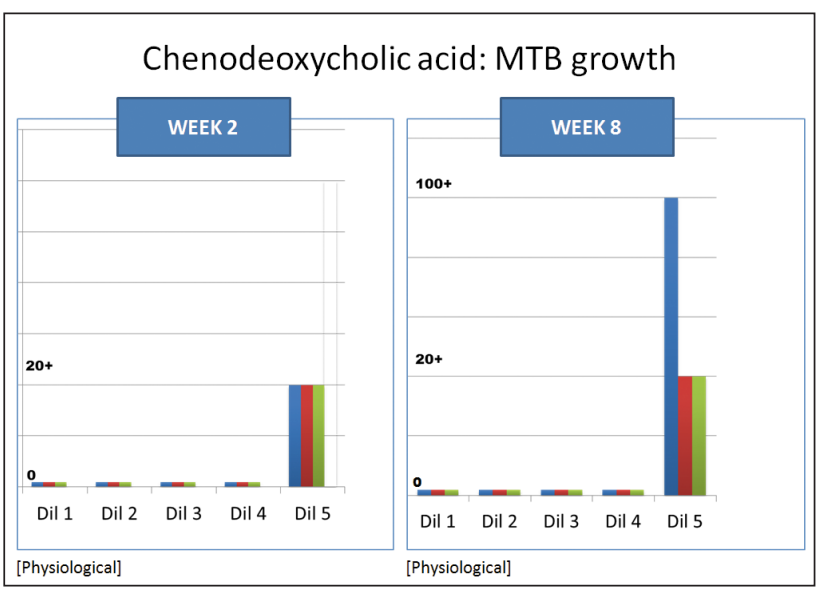

Fig. 1. Chenodeoxycholic acid: MTB growth.

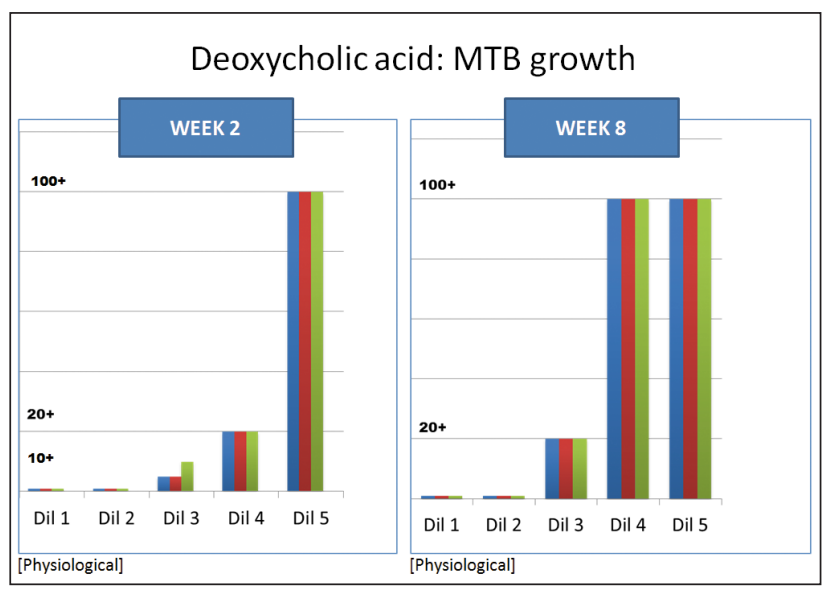

Fig. 2. Deoxycholic acid: MTB growth.

\section{Discussion}

TB of the upper gastrointestinal tract is rare, and isolated TB of the gallbladder very rare, with 5 patients making up the largest case series. ${ }^{5}$ Gallbladder TB usually occurs as an unexpected finding following surgery for benign gallbladder disease ${ }^{5}$ or in the context of immune suppression. ${ }^{6}$ In view of the rarity of gallbladder TB, it is reasonable to consider the gallbladder somewhat resistant to tuberculous infection.

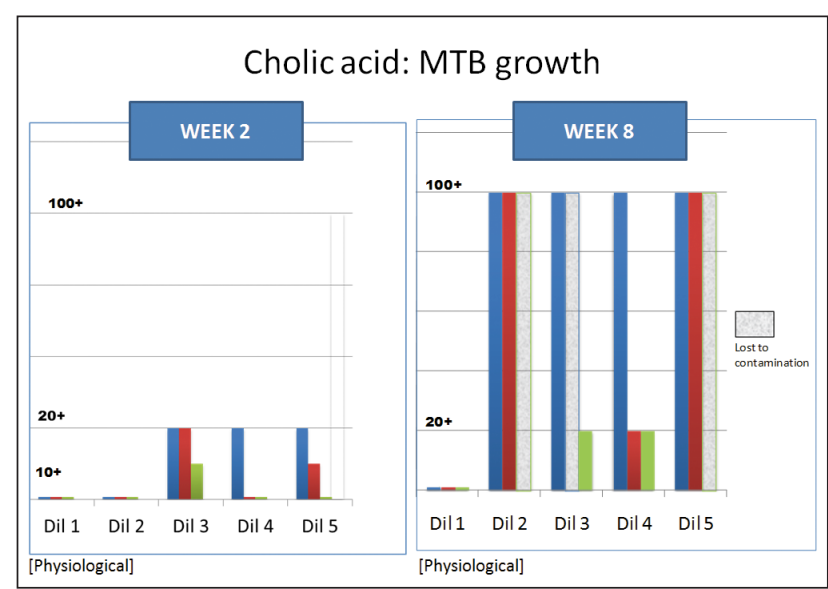

Fig. 3. Cholic acid: MTB growth.

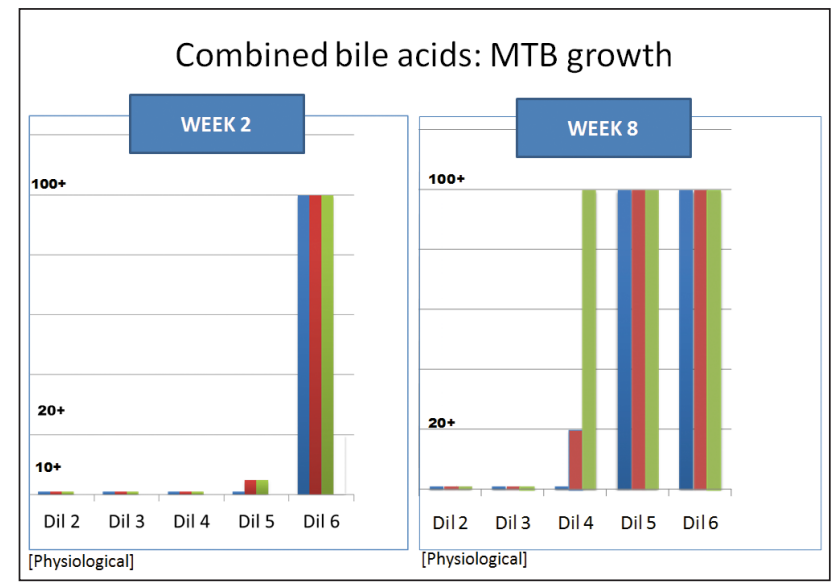

Fig. 4. Combined bile acids: MTB growth.

We have demonstrated that the bile acids chenodeoxycholic acid, deoxycholic acid and cholic acid, alone or in combination, at physiological concentrations, inhibit the growth of MTB in vitro. The anti-mycobacterial effect could be due to the disruption of the lipid-containing mycobacterial cell wall. A concentration-dependent effect was demonstrated and was reproducible. The 8-week results for cholic acid are somewhat surprising, as there seemed to be no effect at $16 \mathrm{mg} / \mathrm{ml}$, but there was an observable inhibitory effect at 
$4 \mathrm{mg} / \mathrm{ml}$. One possible explanation may be related to the difficulties in getting bile salts to dissolve in the LJ medium, which may have resulted in a non-homogeneous solution initially. Similarly, lithocolic acid is the most hydrophobic of the bile acids; reduced solubility in LJ medium may be one explanation for the lack of activity of this bile salt. Alternatively, lithocholic acid may have no intrinsic ability to inhibit mycobacterial growth.

In light of these findings, the interesting possibility is that ursodeoxycholic acid, used as a therapeutic bile acid in cholestatic disorders, may have antimycobacterial effects, but in vitro studies will be required to explore this.

We have demonstrated that bile acids at physiological concentrations inhibit the growth of MTB, raising the possibility that the high concentration of bile acids in the gallbladder and proximal intestine may be protective against $\mathrm{MTB}$, while explaining the predilection of MTB for the terminal ileum and caecum, where bile acid concentrations are at their lowest.

\section{References}

1. Sharma MP, Bhatia V. Abdominal tuberculosis Indian J Med Res 2004;120:305-315.

2. Pettengell KE, Larsen C, Garb M, et al. Gastrointestinal tuberculosis in patients with pulmonary tuberculosis. Q J Med 1990;74:303-308

. Hamilton J, Xie G, Raufman JP, et al. Human cecal bile acids: concentration and spectrum. Am J Physiol Gastrointest Liver Physiol 2007;293:256-263.

4. Nakayama F. Quantititative microanalysis of bile. J Lab Clin Med 1967;69:594-609.

5. Kumar K, Ayub M, Kumar M, et al. M. Tuberculosis of the gallbladder. HPB Surgery 2000;11:401-404. 6. Sobnach S, Van der Linde GD, Kahn D, Bhyat A. Primary tuberculosis of the gallbladder in an HIVpositive patient. S Afr J Surg 2010;48:100.

Accepted 23 February 2012. 